\title{
Contrast-enhanced harmonic endoscopic ultrasonography for the differential diagnosis of pancreatic masses: A systematic review and meta-analysis
}

\author{
YANG LI $^{1 *}$, HAILIN JIN ${ }^{1 *}$, DAN LIAO ${ }^{2}$, BO QIAN ${ }^{2}$, YEIFEI ZHANG ${ }^{2}$, MIN XU $^{2}$ and SHUTANG HAN ${ }^{1}$ \\ ${ }^{1}$ Gastroenterology Endoscopy Center, Affiliated Hospital of Nanjing Medical University of Chinese Medicine \\ (Jiangsu Province Hospital of Chinese Medicine), Nanjing, Jiangsu 210029; ${ }^{2}$ Department of Gastroenterology, \\ Shanghai First People's Hospital of Shanghai Jiaotong University School of Medicine, Shanghai 200011, P.R. China
}

Received August 20, 2018; Accepted July 17, 2019

DOI: $10.3892 /$ mco.2019.1908

\begin{abstract}
Understanding the difference between malignant and benign pancreatic masses is critical in terms of diagnosis, although this is difficult to determine in clinical practice. The contrast-enhanced harmonic endoscopic ultrasound (CH-EUS) technique was introduced in 2010, although, to the best of the authors' knowledge, there has been no systematic review or meta-analysis to date evaluating its diagnostic performance for the differentiation of pancreatic masses. The aim of the present study was to systematically evaluate the diagnostic performance of CH-EUS for the differentiation of pancreatic masses. Search key words and inclusion and exclusion criteria were initially presented. Two independent authors read and extracted the relevant information from the included studies.
\end{abstract}

Correspondence to: Professor Min Xu, Department of Gastroenterology, Shanghai First People's Hospital of Shanghai Jiaotong University School of Medicine, 100 Haining Road, Shanghai 200011, P.R. China

E-mail:xuminmd@163.com

Professor Shutang Han, Gastroenterology Endoscopy Center, Affiliated Hospital of Nanjing Medical University of Chinese Medicine (Jiangsu Province Hospital of Chinese Medicine), 155 Hanzhong Road, Nanjing, Jiangsu 210029, P.R. China

E-mail: shutanghanhst@163.com

"Contributed equally

Abbreviations: EUS, endoscopic ultrasound; EUS-FNA, endoscopic ultrasound-guided fine-needle aspiration; CE-EUS, contrast-enhanced endoscopic ultrasound; PLR, positive likelihood ratio; NLR, negative likelihood ratio; DOR, diagnostic odds ratio; $\mathrm{TP}$, true positive; FP, false positive; $\mathrm{FN}$, false negative; $\mathrm{TN}$, true negative; ROC, receiver operating characteristic; SROC, summary receiver operating characteristic; AUC, area under the curve; QUADAS, Quality Assessment of Diagnostic Accuracy Studies

Key words: contrast-enhanced harmonic endoscopic ultrasound, pancreatic mass, diagnosis
Disagreements were resolved through discussion with another two experienced authors. Metadisc and Stata software were used for the meta-analysis and the evaluation of bias. A total of 16 studies comprising 1,325 patients were included in this meta-analysis. The pooled sensitivity, specificity, positive likelihood ratio, negative likelihood ratio and diagnostic odds ratio of $\mathrm{CH}$-EUS were used to distinguish between malignant and benign tumors, and the values obtained were 93\% [95\% confidence interval (CI): 91-94\%], 84\% (95\% CI: 80-87\%), 5.58 (95\% CI: 3.90-7.97), 0.09 (95\% CI: 0.07-0.11) and 72.56 (95\% CI: 48.93-107.60), respectively. The area under the summary receiver operating characteristic curve was determined to be 0.96 . No publication bias was identified in this meta-analysis. Taken together, these results confirm that $\mathrm{CH}-\mathrm{EUS}$ has a high accuracy rate for distinguishing between benign and malignant pancreatic space-occupying lesions, and it may therefore be used as an effective diagnostic tool for pancreatic masses.

\section{Introduction}

Pancreatic cancer is one of the most aggressive digestive system malignancies, and poses a major threat to human health, representing a major socioeconomic burden worldwide. Moreover, the morbidity and mortality rates of pancreatic cancer continue to rise on a yearly basis (1). The majority of the patients who are initially inflicted with pancreatic cancer do not exhibit any clear clinical symptoms, or only display non-specific symptoms, such as abdominal distention and emesis. Considering that the pancreas is a retroperitoneal organ that is located behind the stomach and duodenum, the application of current imaging techniques is limited (2). As a result, distinguishing pancreatic cancer from other focal pancreatic diseases remains challenging. Defining the properties of pancreatic tumors at an early stage would markedly improve the survival and the quality of life of the patients (2). Contrast-enhanced harmonic endoscopic ultrasound (CH-EUS) is a novel technology that has been used in the diagnosis of pancreatic diseases over the course of the last decade. This method combines EUS with tissue harmonic imaging technology, and overcomes two major shortcomings 
of the traditionally applied method, abdominal Doppler ultrasound: The latter is susceptible to gastrointestinal gas, whereas the former is insensitive to the tiny blood vessels in which the blood flows at low speed (3). With CH-EUS, it is also possible to observe the blood vessels of pancreatic masses in more detail, and it is therefore more accurate in terms of determining pancreatic mass properties (4). To the best of our knowledge, using $\mathrm{CH}$-EUS to differentiate pancreatic malignant from benign masses has only been reported by a few clinical cohort studies $(3,4)$. To date, however, no systematic reviews or meta-analyses have been performed to estimate the diagnostic value of $\mathrm{CH}$-EUS with respect to its ability to differentiate pancreatic malignant tumors from other focal pancreatic diseases. Therefore, a systematic review and meta-analysis has been undertaken in the present study.

\section{Materials and methods}

Literature search. Articles published up to January 2017 in the PubMed, EMBASE, Medline, Web of Science and Cochrane Library databases were searched. The terms used for the search strategy were [('contrast enhanced' OR 'contrast enhancement' OR 'contrast imaging' OR 'contrast agent' OR 'contrast medium') AND 'harmonic'], and ('EUS' OR 'endoscopic ultrasound' OR 'endosonography' OR 'endoscopy ultrasonographic') AND ('pancreatic cancer' or 'pancreatic mass' or 'pancreatitis')].

Inclusion criteria. The criteria for studies to be included in the present systematic review and meta-analysis were as follows: i) $\mathrm{CH}$-EUS was applied to determine the pancreatic mass quality; ii) all patients received a definitive final diagnosis, i.e., malignant tumors were treated with surgical resection or subjected to EUS-guided fine-needle aspiration (EUS-FNA), and benign tumors was followed up for $>6$ months consecutively; iii) the most important four statistics [true positive (TP), false positive (FP), true negative (TN) and false negative $(\mathrm{FN})]$ were extractable from these studies either directly or mediately; (iv) there were no restrictions on the study design or the number of study centers; and (v) although full-text original articles were preferred, abstracts alone were also included in the analysis.

Exclusion criteria. The exclusion criteria were as follows: i) Duplicate studies, case reports, reviews or letters to the editor; ii) statistical data insufficient to construct a $2 \times 2$ diagnostic table; iii) the ultimate diagnoses of patients were not recorded, or the continuous follow-up time of the patients was $<6$ months.

Data collection. Two independent authors (YL and DL) scanned and identified the relevant literature according to the same inclusion and exclusion criteria. When the two interpretations of the data were inconsistent, two other authors (MX and SH) would also critically assess the collection, ensuring that the final result was precise. Detailed information, including the total numbers of patients, the authors' country, patients' mean age, the diameter of the mass, type of study (retrospective/prospective), study center (single/multiple), diagnostic method (qualitative/quantitative), lesion characteristics (solid/cystic), and the TP, FP, TN and FN statistics were extracted for further analysis.

Quality assessment. The Quality Assessment of Diagnostic Accuracy Studies (QUADAS)-2 tool was applied to rate the quality of each included study (5). The QUADAS-2 tool was applied in four phases: The review question was summarized, the tool was tailored to produce review-specific guidance, a flow diagram for the primary study was constructed, and the publication bias and applicability were assessed. This tool allows for more transparent rating of bias and applicability of primary diagnostic accuracy studies (5). This important work was completed by two independent authors (YL and DL), through discussing their results with an additional author $(\mathrm{MX})$ at the time of data collection.

Statistical analysis. Statistical analyses were performed using Metadisc software, version 1.4 (Hospital Universitario Ramon y Cajal) and Stata software, version 14 (StataCorp LP). First, whether the meta-analysis could be accomplished using Metadisc software for the Spearman's test was determined. Subsequently, Metadisc software was used to merge the diagnostic statistical data. Q-test and Chi-squared test were used to determine whether there was any heterogeneity between the results of each study. If $\mathrm{P}<0.1$ or $\mathrm{I}^{2}>50 \%$ was obtained, then heterogeneity was determined to exist, and the random effects model was used to calculate the pooled sensitivity, pooled specificity, pooled negative likelihood ratio (NLR), pooled positive likelihood ratio (PLR), and the diagnostic odds ratio (DOR). Otherwise, the fixed effects model was used to calculate the heterogeneity. Moreover, the factors that cause heterogeneity were identified using meta-regression and subgroup meta-analysis. Finally, the publishing bias of the literature was analyzed by drawing funnel and Egger's publication bias plots using Stata 14.0 software.

\section{Results}

Literature search. A total of 1,440 articles were initially extracted from the PubMed, EMBASE, Medline, Web of Science and Cochrane Library databases. Subsequently, 394 duplicate articles were removed, and clearly irrelevant articles, reviews, case report articles, letters to the editor, and guidelines were also excluded. After reading every abstract and full-text article, 29 articles where Doppler enhancement had been studied, 11 articles on transabdominal ultrasound and 3 articles in which $2 \times 2$ diagnostic tables could not be extracted were excluded, leaving 16 remaining studies that were included in this review (Fig. 1).

Study characteristics. The characteristics of the 16 studies (3,4,6-19), including a total of 1,325 patients, were extracted by two independent authors. Eight studies, including 534 patients, were from the USA and Europe, whereas the remaining 8 studies, including 791 patients, were from Asia. The minimum duration of the included studies was 3 months. Moreover, 12 of the studies also included $>12$ months of follow-up. In addition, of the 16 studies, 10 were retrospective and 6 prospective, whereas 13 were single-center and 3 were multi-center. The majority of the patients included in the 


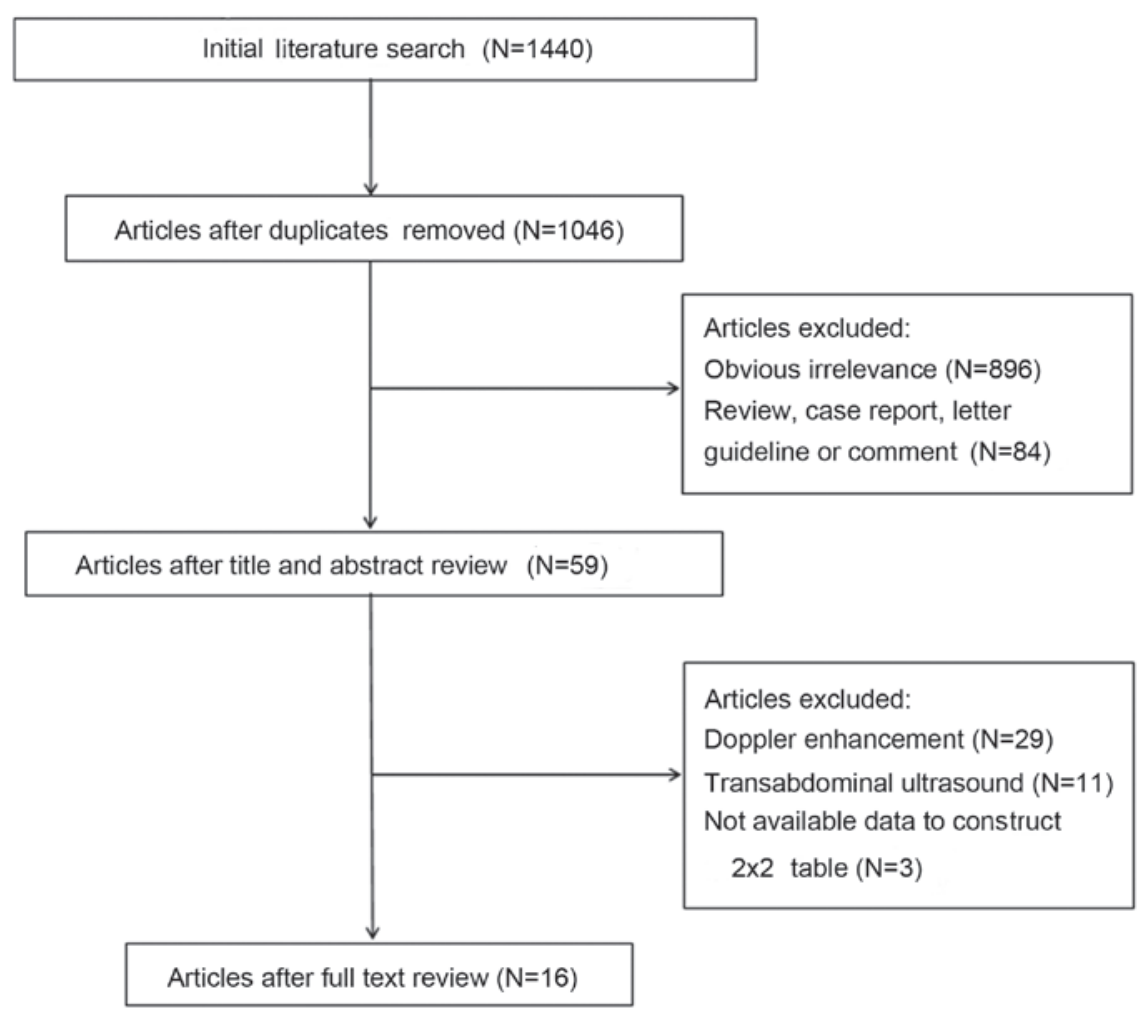

Figure 1. Preferred Reporting Items for Systematic Reviews and Meta-Analyses flow chart for study search.

16 studies were aged $>60$ years; however, Romagnuolo et al (9) failed to report on the mean age of the patients. The diameter of the pancreatic masses ranged from 18.6 to $42.5 \mathrm{~mm}$, and the majority of these masses were solid. The two mainstream ultrasound imaging contrast agents, SonoVue ${ }^{\mathrm{TM}}$ (Bracco) and Sonazoid $^{\mathrm{TM}}$ (GE Healthcare), were used in 15 of the studies. Half of the included studies used a qualitative ultrasound enhancement method, whereas the other half used a quantitative method. A total of 6 studies failed to report on the endosonographer, whereas the diagnoses in the remaining 10 studies were confirmed by more than one endoscopy expert (Table I).

$2 \times 2$ diagnostic tables data and quality of the literature. The four-grid data were registered, and the quality of the included literature was assessed using the QUADAS-2 method (Table II). The risk of bias in the reference index text was not reported by Imazu et al (11). In addition, there was a high risk of bias in flow and timing in the studies of Romagnuolo et al (9), Imazu et al (11) and Iordache et al (18). Overall, all 16 studies were deemed as high-quality.

Diagnostic performance of $C H$-EUS. A Spearman's test was performed, and the Spearman's relative number was identified to be $0.094(\mathrm{P}=0.729)$. Furthermore, on the receiver operating characteristic (ROC) plane, the distribution of the data points did not appear as the characteristic 'shoulder-arm' shape (Fig. 2). Therefore, no threshold effect existed among the 16 studies. The pooled sensitivity, specificity, PLR, NLR and DOR statistics of the CH-EUS analysis were used to distinguish malignant from benign tumors, and these values were determined to be $93 \%$ [95\% confidence interval (CI),
91-94\%], 84\% (95\% CI: 80-87\%), 5.58 (95\% CI: 3.90-7.97), 0.09 (95\% CI: 0.07-0.11) and 72.56 (95\% CI: 48.93-107.60), respectively (Fig. 3A-E). On the summary ROC (SROC) curve, the area under the curve (AUC) was shown to be 0.9611 , which was a significant result, and therefore the malignant tumors were successfully differentiated from the benign ones (Fig. 3F).

Heterogeneity analysis. In order to explore the sources of heterogeneity, subgroup meta-analysis and meta-regression studies were performed, suggesting that the country of origin, total number of patients, the experience of the endoscopy experts, average age, study design, sex, lesion diameter, lesion characteristics, fellow-up duration, the number of study center, diagnostic method and agent type, were not the origin of heterogeneity. Taken together, the results of the subgroup meta-analysis revealed that the diagnostic performance of $\mathrm{CH}$-EUS in terms of differentiating the pancreatic masses was good as well as stable (Table III).

Publication bias. Assessment for the publication bias by funnel plot construction revealed that 2 of the studies lay on the left of the funnel plot, whereas 1 study was positioned to the right (Fig. 4A). Regarding the Egger's publication bias plot, the distribution of spots was linear (Fig. 4B). Based on these results, no publication bias was identified in this meta-analysis.

\section{Discussion}

There are numerous methods that can be employed in order to differentially evaluate pancreatic masses, including 


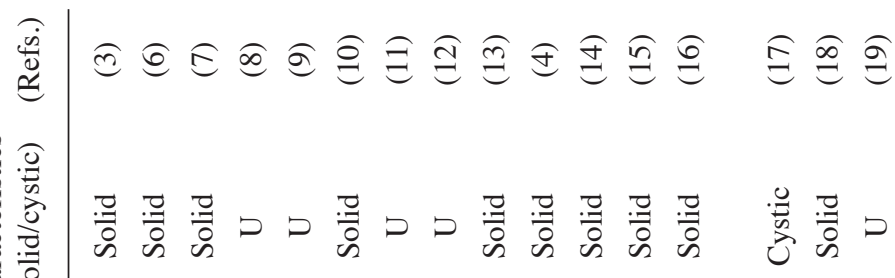

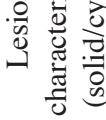

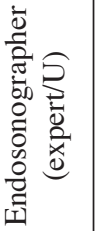

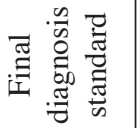

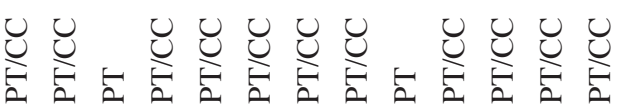

点突

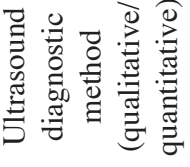

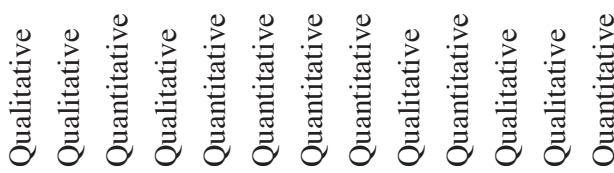

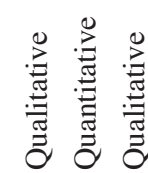

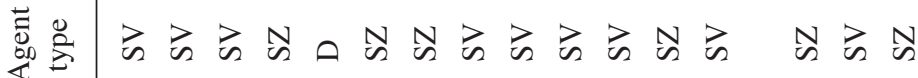

悪

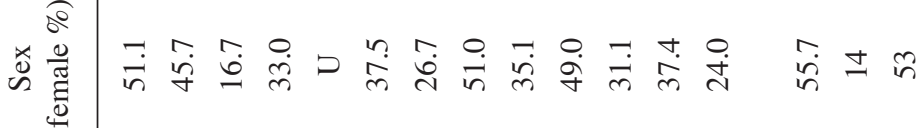

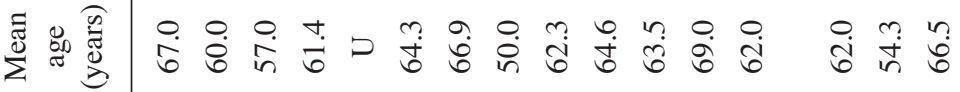

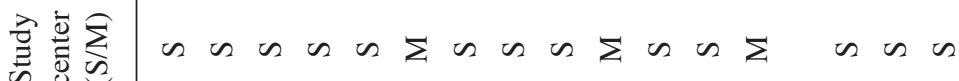

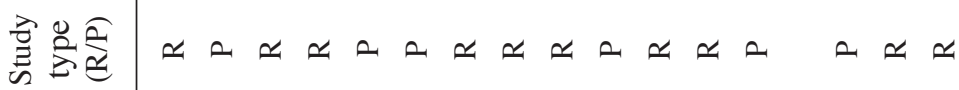

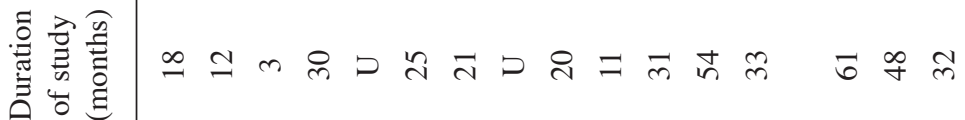

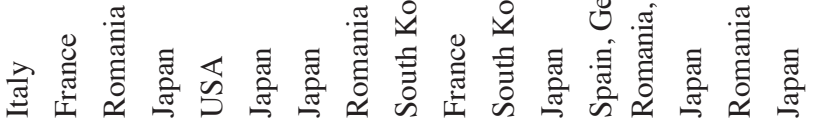

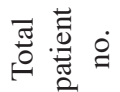

ஓஜ

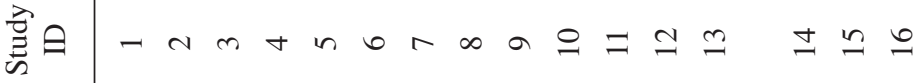

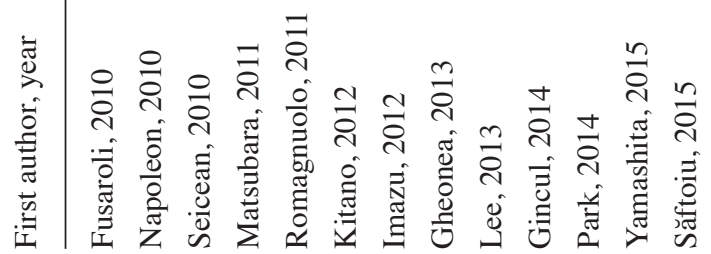

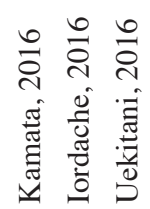

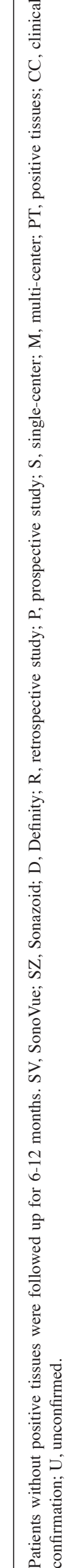


Table II. Derived 2x2 table and QUADAS-2 results.

\begin{tabular}{|c|c|c|c|c|c|c|c|c|c|c|c|}
\hline \multirow[b]{2}{*}{ Study ID } & \multirow[b]{2}{*}{$\mathrm{TP}$} & \multirow[b]{2}{*}{ FP } & \multirow[b]{2}{*}{ FN } & \multirow[b]{2}{*}{$\mathrm{TN}$} & \multicolumn{4}{|c|}{ Risk of bias ${ }^{a}$} & \multicolumn{3}{|c|}{$\begin{array}{l}\text { Concerns regarding } \\
\text { applicability }^{\mathrm{a}}\end{array}$} \\
\hline & & & & & 1 & 2 & 3 & 4 & 1 & 2 & 3 \\
\hline 1 & 49 & 14 & 2 & 25 & $\mathrm{~L}$ & $\mathrm{~L}$ & $\mathrm{~L}$ & $\mathrm{~L}$ & $\mathrm{~L}$ & $\mathrm{~L}$ & $\mathrm{~L}$ \\
\hline 2 & 16 & 2 & 2 & 15 & $\mathrm{~L}$ & $\mathrm{~L}$ & $\mathrm{~L}$ & $\mathrm{~L}$ & $\mathrm{~L}$ & $\mathrm{~L}$ & $\mathrm{~L}$ \\
\hline 3 & 12 & 1 & 3 & 11 & $\mathrm{~L}$ & $\mathrm{~L}$ & $\mathrm{~L}$ & $\mathrm{~L}$ & $\mathrm{~L}$ & $\mathrm{~L}$ & $\mathrm{~L}$ \\
\hline 4 & 46 & 3 & 2 & 40 & $\mathrm{~L}$ & $\mathrm{U}$ & $\mathrm{L}$ & $\mathrm{L}$ & $\mathrm{L}$ & $\mathrm{L}$ & $\mathrm{L}$ \\
\hline 5 & 9 & 0 & 0 & 2 & $\mathrm{~L}$ & $\mathrm{~L}$ & $\mathrm{~L}$ & $\mathrm{H}$ & $\mathrm{L}$ & $\mathrm{L}$ & $\mathrm{L}$ \\
\hline 6 & 194 & 9 & 10 & 64 & $\mathrm{~L}$ & $\mathrm{~L}$ & $\mathrm{~L}$ & $\mathrm{~L}$ & $\mathrm{~L}$ & $\mathrm{~L}$ & $\mathrm{~L}$ \\
\hline 7 & 22 & 0 & 0 & 8 & $\mathrm{~L}$ & $\mathrm{U}$ & $\mathrm{L}$ & $\mathrm{H}$ & $\mathrm{L}$ & $\mathrm{L}$ & $\mathrm{L}$ \\
\hline 8 & 30 & 2 & 2 & 17 & $\mathrm{~L}$ & $\mathrm{~L}$ & $\mathrm{~L}$ & $\mathrm{~L}$ & $\mathrm{~L}$ & $\mathrm{~L}$ & $\mathrm{~L}$ \\
\hline 9 & 28 & 1 & 2 & 6 & $\mathrm{~L}$ & $\mathrm{~L}$ & $\mathrm{~L}$ & $\mathrm{~L}$ & $\mathrm{~L}$ & $\mathrm{~L}$ & $\mathrm{~L}$ \\
\hline 10 & 66 & 2 & 3 & 29 & $\mathrm{~L}$ & $\mathrm{~L}$ & $\mathrm{~L}$ & $\mathrm{~L}$ & $\mathrm{~L}$ & $\mathrm{~L}$ & $\mathrm{~L}$ \\
\hline 11 & 57 & 9 & 5 & 19 & $\mathrm{~L}$ & $\mathrm{~L}$ & $\mathrm{~L}$ & $\mathrm{~L}$ & $\mathrm{~L}$ & $\mathrm{~L}$ & $\mathrm{~L}$ \\
\hline 12 & 102 & 11 & 7 & 27 & $\mathrm{~L}$ & $\mathrm{~L}$ & $\mathrm{~L}$ & $\mathrm{~L}$ & $\mathrm{~L}$ & $\mathrm{~L}$ & $\mathrm{~L}$ \\
\hline 13 & 98 & 4 & 14 & 51 & $\mathrm{~L}$ & $\mathrm{~L}$ & $\mathrm{~L}$ & $\mathrm{~L}$ & $\mathrm{~L}$ & $\mathrm{~L}$ & $\mathrm{~L}$ \\
\hline 14 & 29 & 10 & 1 & 30 & $\mathrm{~L}$ & $\mathrm{~L}$ & $\mathrm{~L}$ & $\mathrm{~L}$ & $\mathrm{~L}$ & $\mathrm{~L}$ & $\mathrm{~L}$ \\
\hline 15 & 17 & 6 & 2 & 25 & $\mathrm{~L}$ & $\mathrm{~L}$ & $\mathrm{~L}$ & $\mathrm{H}$ & $\mathrm{L}$ & $\mathrm{L}$ & $\mathrm{L}$ \\
\hline 16 & 32 & 0 & 8 & 9 & $\mathrm{~L}$ & $\mathrm{~L}$ & $\mathrm{~L}$ & $\mathrm{~L}$ & $\mathrm{~L}$ & $\mathrm{~L}$ & $\mathrm{~L}$ \\
\hline
\end{tabular}

a 1 , patient selected; 2 , index text; 3 , reference standard; 4, flow and timing. L, low risk; H, high risk; U, unclear risk; TP, true positive; FP, false positive; FN, false negative; TN, true negative; QUADAS, Quality Assessment of Diagnostic Accuracy Studies.

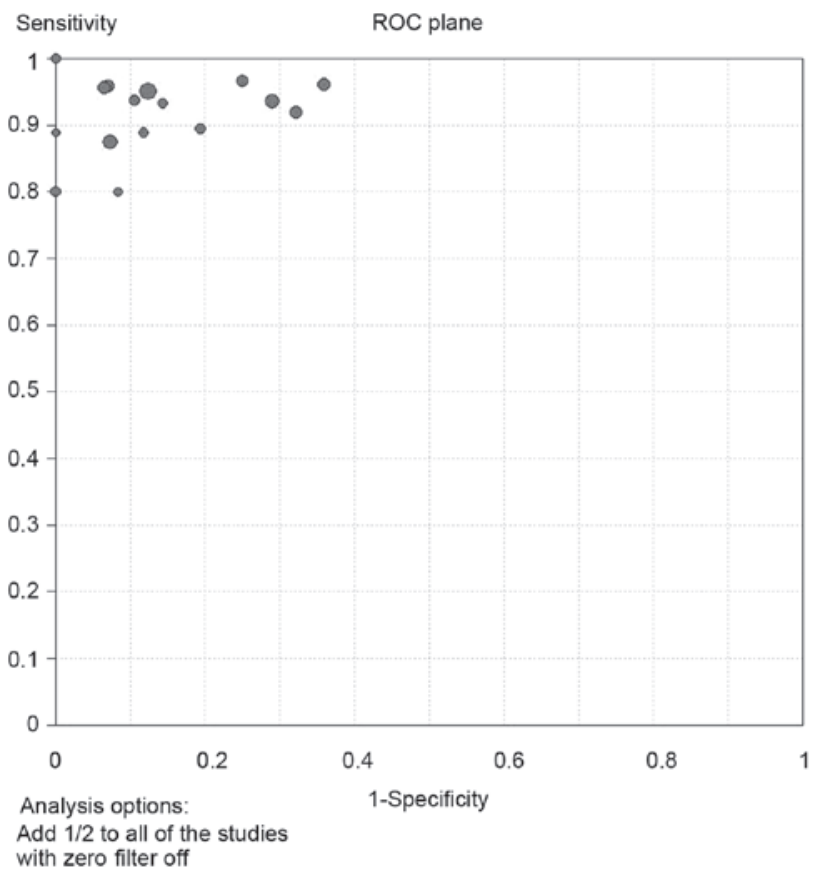

Figure 2. ROC plane. ROC, receiver operating characteristic.

transabdominal ultrasound, computed tomography (CT) and magnetic resonance imaging, among others. However, the sensitivity and specificity of these traditional diagnostic methods are low, particularly when the diameter of the tumor is $<2 \mathrm{~cm}$ (20). EUS has been applied in the clinic for decades, with some success. It is able to overcome the gas interference associated with transabdominal ultrasound; therefore, EUS has been established as a suitable method for differentiating malignant from benign pancreatic masses. However, it is highly affected by the experience of the endosonographer as regards EUS image acquisition (21). EUS-FNA, a technique derived from EUS, is a new diagnostic method by which tissue samples of pancreatic masses are obtained for pathology diagnosis. EUS-FNA has achieved diagnostic sensitivity and specificity rates of up to 95 and $100 \%$, respectively (22). However, EUS-FNA is invasive, and the procedure is difficult and complicated. If the procedure is not successful in obtaining positive tissue, repeat EUS-FNA is deemed necessary. Contrast-enhanced EUS (CE-EUS) combines enhanced ultrasonography with endoscopy. After injecting a contrast agent, the blood flow signal of pancreatic tumors was found to be markedly improved. Sakamoto et al (23) demonstrated that the sensitivity of the diagnosis of pancreatic cancer was $94.4 \%$ with CE-EUS, which was a significant improvement compared with enhanced CT. However, as an earlier established enhancement type, Doppler enhancement is not very sensitive to the small vessels of the pancreas and, therefore, induces the so-called 'flower' phenomenon, which arises from the fake blood flow signal. The emergence of CH-EUS, however, has made up for these defects. CH-EUS was reported by Dietrich et al as early as 2005 (24). This group first used harmonic enhancement, another more efficient enhancement mode, to observe the abdominal vessels. $\mathrm{CH}$-EUS utilizes a selective harmonic detection element that, when mounted at the front of the endoscope, is able to detect the non-linear signal of the intravascular microbubble produced by the contrast agent, while filtering out the differential signal derived from the tissue and avoiding hypo-enhancement with the heterogeneous pattern of the pancreatic mass lesion. Upon $\mathrm{CH}$-EUS imaging, pancreatic 
A

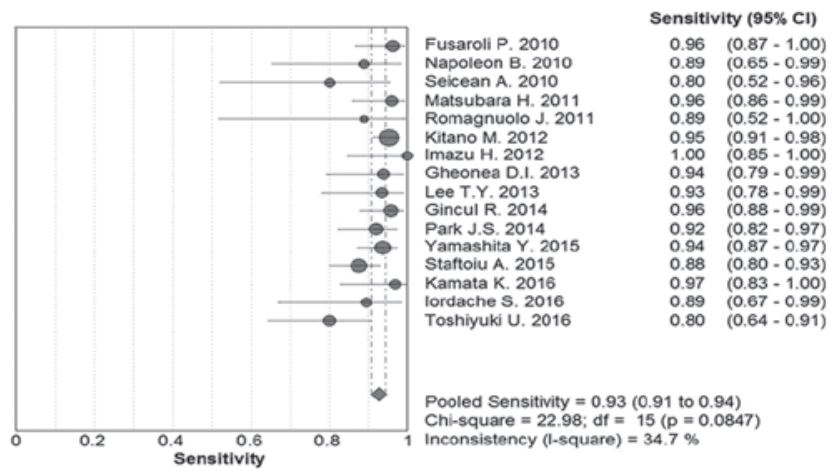

Analysis Options:
Add $1 / 2$ to all cells of the studies with zero
Filter OFF

C
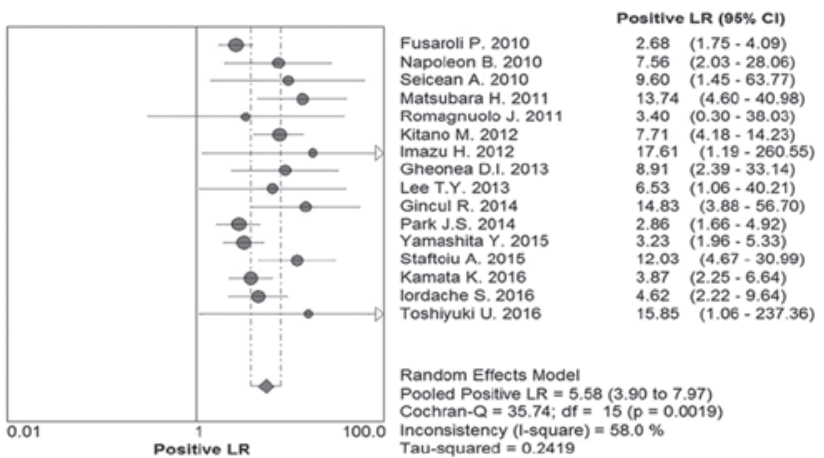

Analysis Options:
Add $1 / 2$ to all cells of the studies with zero
Filter OFF

$\mathrm{E}$

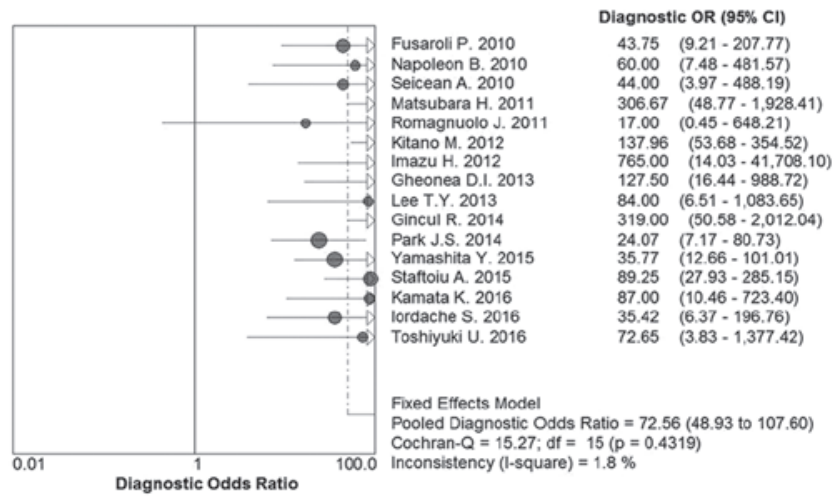

Analysis Options:
Add $1 / 2$ to all cells of the studies with zero Filter OFF
B

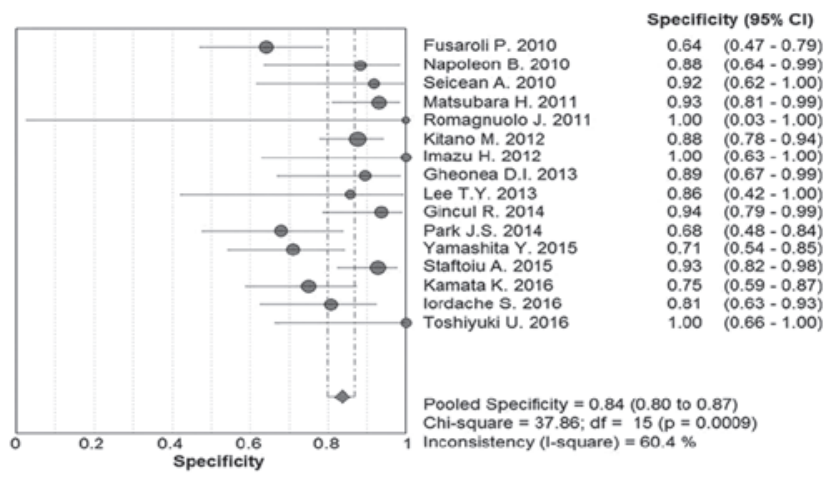

Analysis Options:

Add $1 / 2$ to all cells of the studies with zero
Filter OFF

D

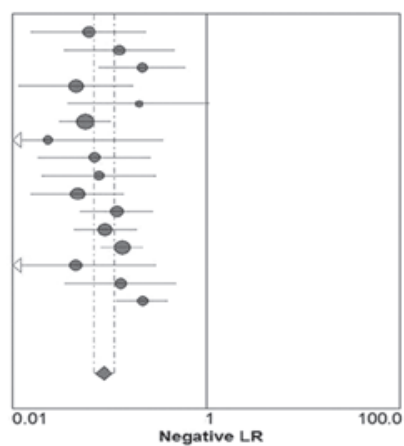

Fusaroli P. 2010 Napoleon B. 2010 Matsubara H. 2011 Romagnuolo J. 201 Imazu H. 2012 Gheonea D.I. 201 Lee T.Y. 2013
Gincul R. 2014 Park J.S. 2014 Yamashita Y. 2015
Staftoiu A. 2015 Kamata K. 2016 lordache S. 2016
Toshiyuki U. 2016

Negative LR (95\% C $0.06 \quad(0.02-0.24)$ $\begin{array}{ll}0.06 & (0.02-0.24) \\ 0.13 & (0.03-0.47 \\ 0.22 & (0.08-0.61) \\ 0.04 & (0.01-0.17)\end{array}$ $0.22 \quad(0.08-0.61)$ $0.04 \quad(0.01-0.17$ $\begin{array}{ll}0.06 & (0.03-0.10) \\ 0.02 & (0.00-0.36)\end{array}$ $\begin{array}{ll}0.02 & (0.00-0.36) \\ 0.07 & (0.02-0.27)\end{array}$ $\begin{array}{ll}0.07 & (0.02-0.27) \\ 0.08 & (0.02-0.31) \\ 0.05 & (0.02-0.14)\end{array}$ $0.12(0.05-0.29)$ $0.09 \quad(0.04-0.19)$ $0.13 \quad(0.08-0.22$ $\begin{array}{ll}0.04 & (0.01-0.31 \\ 0.13 & (0.03-0.49)\end{array}$ Fixed Effects Model Pooled Negative $L R=0.09(0.07$ to 0.11$)$ Cochran- $Q=22.55 ;$ df $=15(p=0.0942)$

Analysis Options: Add $1 / 2$ to all cells of the studies with zero
Filter OFF

F

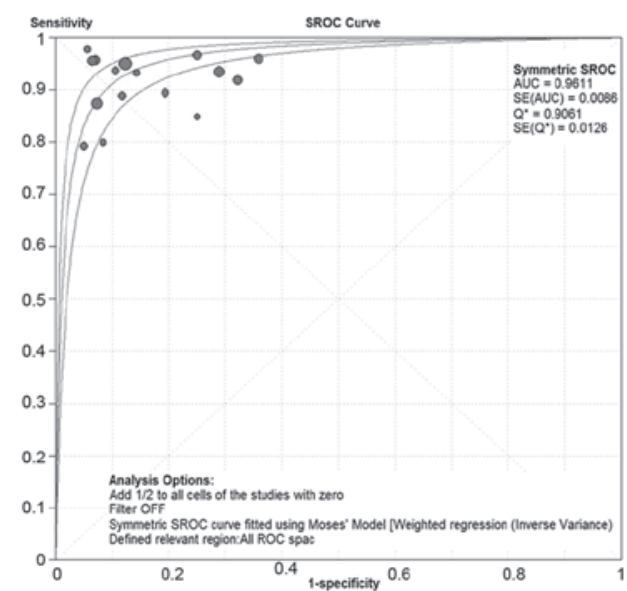

Figure 3. Diagnostic performance of CH-EUS was performed using Metadisc software, version 1.4 (Hospital Universitario Ramon y Cajal). (A) Forest plot of pooled sensitivity of CH-EUS; (B) forest plot of pooled specificity of CH-EUS; (C) pooled PLR of CH-EUS; (D) pooled NLR of CH-EUS; (E) pooled DOR of CH-EUS; (F) SROC curve of CH-EUS. CH-EUS, contrast-enhanced harmonic endoscopy ultrasound; PLR, positive likelihood ratio; NLR, negative likelihood ratio; DOR, diagnostic odds ratio; SROC, summary receiver operating characteristic.

malignant tissue exhibits low and uneven vascular enhancement, making it easy to distinguish malignant from benign tissue. As a result, the accuracy of CH-EUS in terms of diagnosis of pancreatic cancer was found to be markedly improved $(25,26)$.

To the best of the authors' knowledge, this is the first systematic review and meta-analysis to estimate the diagnostic performance of $\mathrm{CH}$-EUS for the differentiation of pancreatic masses since 2010, when CH-EUS was introduced by Fusaroli et al (3). In this meta-analysis, the characteristic 'shoulder-arm' shape of the data point distribution was found to be lacking on the ROC plane. Of note, no threshold was set in the sensitivity and specificity among the 16 included studies. In the present review, the pooled sensitivity was $>90 \%$, and the pooled specificity was also $>80 \%$. It is well established that the likelihood ratios (PLR and NLR) and DOR are reflections of the composite index of sensitivity and specificity, which are more sensitive compared with sensitivity and specificity 
Table III. Predefined subgroup analysis of indices and subsequent meta-regression on DOR.

\begin{tabular}{|c|c|c|c|c|c|c|c|c|c|}
\hline Subgroup & $\begin{array}{c}\text { Study } \\
\text { numbers }\end{array}$ & $\begin{array}{l}\text { Pooled sensitivity } \\
\text { (95\% CI) }\end{array}$ & $\mathrm{I}^{2} \%$ & $\begin{array}{l}\text { Pooled specificity } \\
\text { (95\% CI })\end{array}$ & $\mathrm{I}^{2} \%$ & $\begin{array}{l}\text { Diagnostic odds } \\
\text { ratio }(95 \% \mathrm{CI})\end{array}$ & $\mathrm{I}^{2} \%$ & $\begin{array}{l}\text { RDOR } \\
(95 \% \mathrm{CI})\end{array}$ & P-value \\
\hline \multicolumn{10}{|l|}{ Area } \\
\hline Europe/America & 8 & $0.91(0.87-0.94)$ & 11.8 & $0.85(0.79-0.89)$ & 59.6 & $71.60(38.55-132.95)$ & 0 & $1.3(0.45-3.81)$ & 0.60 \\
\hline Asian & 8 & $0.94(0.91-0.95)$ & 47.0 & $0.83(0.77-0.87)$ & 65.2 & $73.30(43.97-122.20)$ & 33.3 & & \\
\hline \multicolumn{10}{|l|}{ Total patients } \\
\hline$\geq 85$ & 7 & $0.93(0.91-0.95)$ & 26.8 & $0.83(0.78-0.87)$ & 78.4 & $78.60(39.13-157.91)$ & 50.5 & $0.74(0.24-2.24)$ & 0.56 \\
\hline$<85$ & 9 & $0.90(0.85-0.94)$ & 35.9 & $0.85(0.78-0.90)$ & 19.5 & $67.43(31.13-146.05)$ & 0 & & \\
\hline \multicolumn{10}{|l|}{ Duration, months } \\
\hline$\geq 28$ & 7 & $0.94(0.91-0.95)$ & 47 & $0.83(0.77-0.87)$ & 65.2 & $73.30(43.97-122.20)$ & 33.3 & $1.60(0.76-3.40)$ & 0.20 \\
\hline$<28$ & 7 & $0.94(0.91-0.95)$ & 47 & $0.83(0.77-0.87)$ & 65.2 & $73.30(43.97-122.20)$ & 33.3 & & \\
\hline Unconfirmed & 2 & $0.94(0.91-0.95)$ & 47 & $0.83(0.77-0.87)$ & 65.2 & $73.30(43.97-122.20)$ & 33.3 & & \\
\hline \multicolumn{10}{|l|}{ Study center } \\
\hline Single & 13 & $0.92(0.90-0.95)$ & 25.9 & $0.80(0.75-0.84)$ & 55.8 & $54.42(33.53-88.31)$ & 0 & $2.32(0.83-6.45)$ & 0.10 \\
\hline Multiple & 3 & $0.93(0.90-0.95)$ & 70.0 & $0.91(0.85-0.95)$ & 0 & $130.36(66.11-257.04)$ & 0 & & \\
\hline \multicolumn{10}{|l|}{ Design } \\
\hline Retrospective & 10 & $0.92(0.89-0.95)$ & 39.1 & $0.80(0.74-0.85)$ & 64.4 & $52.93(31.52-88.87)$ & 0 & $1.98(0.75-5.21)$ & 0.15 \\
\hline Prospective & 6 & $0.93(0.90-0.95)$ & 37.3 & $0.88(0.82-0.92)$ & 35.1 & $109.86(59.68-202.24)$ & 0 & & \\
\hline \multicolumn{10}{|l|}{ Mean age, years } \\
\hline$\geq 60$ & 12 & $0.93(0.91-0.95)$ & 48.7 & $0.83(0.79-0.87)$ & 72 & $77.82(50.39-120.20)$ & 25.5 & $0.51(0.16-1.60)$ & 0.22 \\
\hline$<60$ & 3 & $0.89(0.81-0.95)$ & 0 & $0.86(0.76-0.93)$ & 0 & $55.62(20.59-150.19)$ & 0 & & \\
\hline Unconfirmed & 1 & $0.89(0.52-1.00)$ & 0 & $1.00(0.03-1.00)$ & 0 & $17.00(0.45-648.21)$ & & & \\
\hline \multicolumn{10}{|l|}{ Sex (female \%) } \\
\hline$\geq 50$ & 4 & $0.89(0.81-0.95)$ & 0 & $0.86(0.76-0.93)$ & 0 & $55.62(20.59-150.19)$ & 0 & $0.74(0.21-2.56)$ & 0.61 \\
\hline$<50$ & 11 & $0.93(0.91-0.95)$ & 29.2 & $0.86(0.82-0.89)$ & 53.8 & $75.15(48.95-115.38)$ & 28.2 & & \\
\hline Unconfirmed & 1 & $0.89(0.52-1.00)$ & 0 & $1.00(0.03-1.00)$ & 0 & $17.00(0.45-648.21)$ & & & \\
\hline \multicolumn{10}{|l|}{ Agent type } \\
\hline SonoVue & 9 & $0.91(0.88-0.94)$ & 1.1 & $0.83(0.77-0.87)$ & 62.5 & $60.69(35.52-103.68)$ & 0 & $1.54(0.64-3.70)$ & 0.31 \\
\hline Sonazoid & 6 & $0.94(0.91-0.96)$ & 61.2 & $0.84(0.79-0.89)$ & 68.8 & $94.23(52.04-170.61)$ & 24.7 & & \\
\hline Others & 1 & $0.89(0.52-1.00)$ & 0 & $1.00(0.03-1.00)$ & 0 & $17.00(0.45-648.21)$ & & & \\
\hline \multicolumn{10}{|l|}{ Diagnostic method } \\
\hline Qualitative & 8 & $0.93(0.89-0.95)$ & 29.4 & $0.77(0.71-0.83)$ & 64.5 & $52.62(30.29-91.43)$ & 0 & $1.91(0.65-5.59)$ & 0.22 \\
\hline Quantitative & 8 & $0.93(0.90-0.95)$ & 46.3 & $0.90(0.85-0.93)$ & 0 & $101.89(57.81-179.60)$ & 0 & & \\
\hline \multicolumn{10}{|l|}{ Endosonographer } \\
\hline Expert & 10 & $0.94(0.92-0.96)$ & 0 & $0.80(0.75-0.84)$ & 62.0 & $69.14(43.23-110.58)$ & 21.3 & $0.61(0.17-2.15)$ & 0.41 \\
\hline Unconfirmed & 6 & $0.88(0.84-0.92)$ & 13.3 & $0.90(0.85-0.95)$ & 10.0 & $80.57(38.97-166.55)$ & 0 & & \\
\hline \multicolumn{10}{|c|}{ Lesion characteristics } \\
\hline Solid & 10 & $0.93(0.91-0.95)$ & 18.5 & $0.82(0.78-0.86)$ & 64.1 & $63.33(41.17-97.43)$ & 12.8 & $1.46(0.74-2.90)$ & 0.25 \\
\hline Cystic & 1 & $0.97(0.83-1.00)$ & 0 & $0.75(0.59-0.87)$ & 0 & $87.00(10.46-723.40)$ & & & \\
\hline Unconfirmed & 5 & $0.91(0.86-0.95)$ & 62.6 & $0.94(0.86-0.98)$ & 0 & $145.34(46.35-455.78)$ & 0 & & \\
\hline \multicolumn{10}{|c|}{ Lesion diameter, $\mathrm{mm}$} \\
\hline$\geq 30$ & 10 & $0.92(0.90-0.94)$ & 56 & $0.86(0.82-0.90)$ & 52.7 & $87.27(53.55-142.22)$ & 0 & $0.72(0.4-12.8)$ & 0.24 \\
\hline$<30$ & 3 & $0.95(0.90-0.98)$ & 0 & $0.80(0.69-0.88)$ & 82.6 & $79.39(26.43-238.43)$ & 39.9 & & \\
\hline Unconfirmed & 3 & $0.92(0.85-0.96)$ & 0 & $0.78(0.67-0.87)$ & 40.8 & $37.31(15.88-87.65)$ & 0 & & \\
\hline
\end{tabular}

RDOR, relative diagnostic odds ratio; $\mathrm{CI}$, confidence interval.

alone. In the present review, the pooled PLR, NLR and DOR also revealed that $\mathrm{CH}$-EUS performed well in the diagnosis of pancreatic masses. The area under SROC, identified to be 0.96 , was very close to 1 . Furthermore, it was also demonstrated that the country of origin, number of patients, study design, selection of contrast enhancement agent, experience of the endosonographer, lesion characteristics, ultrasound evaluation method, and an additional 9 factors, did not have a significant impact on the diagnostic performance of CH-EUS in terms of the differentiation of the pancreatic mass by subgroup meta-analysis and meta-regression. Finally, only articles published in English were searched in the PubMed, EMBASE, Medline, Web of Science and Cochrane Library databases, and the overall quality of a number of the studies 
A

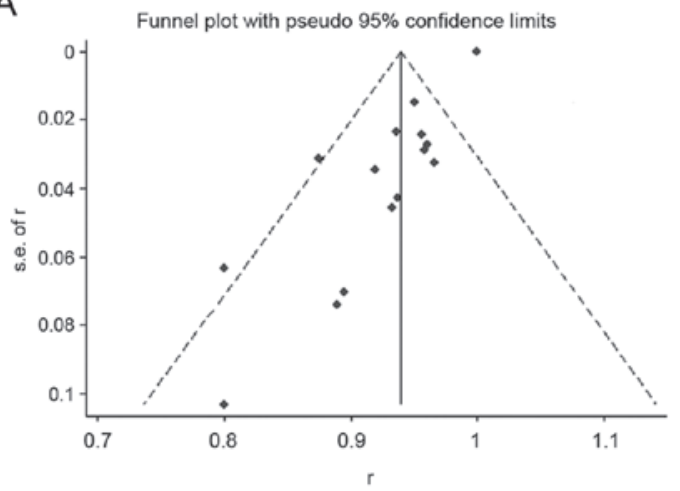

B

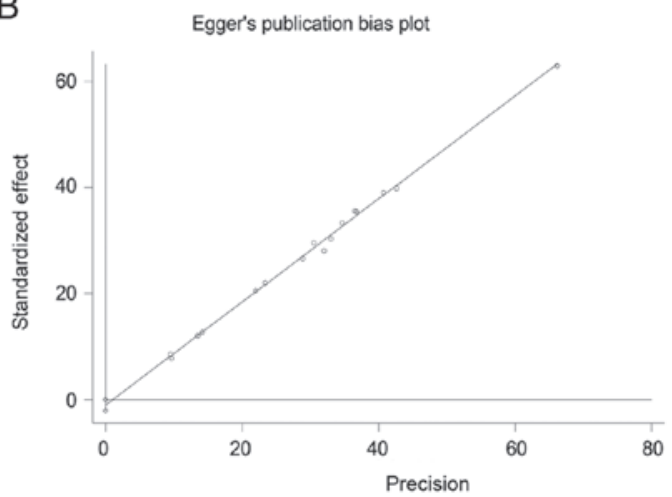

Figure 4. Publication bias was analyzed by drawing funnel plots and Egger's publication bias plots using Stata 14.0 software (StataCorp LP). (A) Evaluation of publication bias by funnel plot; (B) Egger's publication bias plot.

identified was not high. However, the literature publication bias of these included 16 studies was acceptable. Based on the above findings, and it was possible to conclude that CH-EUS performed well as a technique in the differential diagnosis of pancreatic masses.

In conclusion, the present study demonstrated that CH-EUS is characterized by high accuracy for differentiating between benign and malignant pancreatic space-occupying lesions, and it also has the advantages of being non-invasive and cost-effective. Therefore, it may prove to be an effective method for the identification of benign and malignant pancreatic lesions in the future.

\section{Acknowledgements}

Not applicable.

\section{Funding}

The present study was supported by an innovation fund of the Jiangsu Hospital of Chinese Medicine (grant no. Y2018CX57) and the second batch of scientific research special projects for the construction of the National TCM Clinical Research Base in 2015 (grant no. JDZX2015086).

\section{Availability of data and materials}

All data generated or analyzed during the present study are included in this published article.

\section{Authors' contributions}

YL and DL scanned and identified the relevant literature according to the inclusion and exclusion criteria. YL, HJ, BQ and YZ wrote and modified parts of the manuscript. MX and SH conceived the study and conducted a critical pre-submission review of the manuscript. All the authors have read and approved the final version of the manuscript for publication.

\section{Ethics approval and consent to participate}

Not applicable.

\section{Patient consent for publication}

Not applicable.

\section{Competing interests}

The authors declare that they have no competing interests.

\section{References}

1. Torre LA, Bray F, Siegel RL, Ferlay J, Lortet-Tieulent J and Jemal A: Global cancer statistics, 2012. CA Cancer J Clin 65: 87-108, 2015

2. Hackeng WM, Hruban RH, Offerhaus GJ and Brosens LA: Surgical and molecular pathology of pancreatic neoplasms. Diagn Pathol 11: 47, 2016.

3. Fusaroli P, Spada A, Mancino MG and Caletti G: Contrast harmonic echo-endoscopic ultrasound improves accuracy in diagnosis of solid pancreatic masses. Clin Gastroenterol Hepatol 8: 629-634.e1-e2, 2010.

4. Gincul R, Palazzo M, Pujol B, Tubach F, Palazzo L, Lefort C, Fumex F, Lombard A, Ribeiro D, Fabre M, et al: Contrast-harmonic endoscopic ultrasound for the diagnosis of pancreatic adenocarcinoma: A prospective multicenter trial. Endoscopy 46: 373-379, 2014.

5. Whiting PF, Rutjes AW, Westwood ME, Mallett S, Deeks JJ, Reitsma JB, Leeflang MM, Sterne JA and Bossuyt PM; QUADAS-2 Group: QUADAS-2: A revised tool for the quality assessment of diagnostic accuracy studies. Ann Intern Med 155: 529-536, 2011.

6. Napoleon B, Alvarez-Sanchez MV, Gincoul R, Pujol B, Lefort C, Lepilliez V, Labadie M, Souquet JC, Queneau PE, Scoazec JY, et al: Contrast-enhanced harmonic endoscopic ultrasound in solid lesions of the pancreas: Results of a pilot study. Endoscopy 42: 564-570, 2010.

7. Seicean A, Badea R, Stan-Iuga R, Mocan T, Gulei I and Pascu O: Quantitative contrast-enhanced harmonic endoscopic ultrasonography for the discrimination of solid pancreatic masses. Ultraschall Med 31: 571-576, 2010.

8. Matsubara H, Itoh A, Kawashima H, Kasugai T, Ohno E, Ishikawa T, Itoh Y, Nakamura Y, Hiramatsu T, Nakamura M, et al: Dynamic quantitative evaluation of contrast-enhanced endoscopic ultrasonography in the diagnosis of pancreatic diseases. Pancreas 40: 1073-1079, 2011.

9. Romagnuolo J, Hoffman B, Vela S, Hawes R and Vignesh S: Accuracy of contrast-enhanced harmonic EUS with a secondgeneration perflutren lipid microsphere contrast agent (with video). Gastrointest Endosc 73: 52-63, 2011.

10. Kitano M, Kudo M, Yamao K, Takagi T, Sakamoto H, Komaki T, Kamata K, Imai H, Chiba Y, Okada M, et al: Characterization of small solid tumors in the pancreas: The value of contrast-enhanced harmonic endoscopic ultrasonography. Am J Gastroenterol 107: 303-310, 2012. 
11. Imazu H, Kanazawa K, Mori N, Ikeda K, Kakutani H, Sumiyama K, Hino S, Ang TL, Omar S and Tajiri H: Novel quantitative perfusion analysis with contrast-enhanced harmonic EUS for differentiation of autoimmune pancreatitis from pancreatic carcinoma. Scand J Gastroenterol 47: 853-860, 2012.

12. Gheonea DI, Streba CT, Ciurea T and Săftoiu A: Quantitative low mechanical index contrast-enhanced endoscopic ultrasound for the differential diagnosis of chronic pseudotumoral pancreatitis and pancreatic cancer. BMC Gastroenterol 13: 2, 2013.

13. Lee TY, Cheon YK and Shim CS: Clinical role of contrastenhanced harmonic endoscopic ultrasound in differentiating solid lesions of the pancreas: A single-center experience in Korea. Gut Liver 7: 599-604, 2013.

14. Park JS, Kim HK, Bang BW, Kim SG, Jeong S and Lee DH: Effectiveness of contrast-enhanced harmonic endoscopic ultrasound for the evaluation of solid pancreatic masses. World J Gastroenterol 20: 518-524, 2014.

15. Yamashita Y, Kato J, Ueda K, Nakamura Y, Kawaji Y, Abe H, Nuta J, Tamura T, Itonaga M, Yoshida T, et al: Contrast-enhanced endoscopic ultrasonography for pancreatic tumors. Biomed Res Int 2015: 491782, 2015

16. Săftoiu A, Vilmann P, Dietrich CF, Iglesias-Garcia J, Hocke M, Seicean A, Ignee A, Hassan H, Streba CT, Ioncică AM, et al: Quantitative contrast-enhanced harmonic EUS in differential diagnosis of focal pancreatic masses (with videos). Gastrointest Endosc 82: 59-69, 2015

17. Kamata K, Kitano M, Omoto S, Kadosaka K, Miyata T, Yamao K, Imai H, Sakamoto H, Harwani Y, Chikugo T, et al: Contrast-enhanced harmonic endoscopic ultrasonography for differential diagnosis of pancreatic cysts. Endoscopy 48: 35-41, 2016.

18. Iordache S, Costache MI, Popescu CF, Streba CT, Cazacu S and Săftoiu A: Clinical impact of EUS elastography followed by contrast-enhanced EUS in patients with focal pancreatic masses and negative EUS-guided FNA. Med Ultrason 18: $18-24,2016$
19. Uekitani T, Kaino S, Harima H, Suenaga S, Sen-Yo M and Sakaida I: Efficacy of contrast-enhanced harmonic endoscopic ultrasonography in the diagnosis of pancreatic ductal carcinoma. Saudi J Gastroenterol 22: 198-202, 2016.

20. Krishna SG and Lee JH: Endosonography in solid and cystic pancreatic tumors. J Interv Gastroenterol 1: 193-201, 2011.

21. Kim J: Endoscopic ultrasound-guided treatment of pancreatic cystic and solid masses. Clin Endosc 48: 308-311, 2015.

22. Eloubeidi MA, Jhala D, Chhieng DC, Chen VK, Eltoum I, Vickers S, Mel Wilcox C and Jhala N: Yield of endoscopic ultrasound-guided fine-needle aspiration biopsy in patients with suspected pancreatic carcinoma. Cancer 99: 285-292, 2003.

23. Sakamoto H, Kitano M, Suetomi Y, Maekawa K, Takeyama Y and Kudo M: Utility of contrast-enhanced endoscopic ultrasonography for diagnosis of small pancreatic carcinomas. Ultrasound Med Biol 34: 525-532, 2008.

24. Dietrich CF, Ignee A and Frey H: Contrast-enhanced endoscopic ultrasound with low mechanical index: A new technique. Z Gastroenterol 43: 1219-1223, 2005

25. Wu RI, Yoon WJ, Brugge WR, Mino-Kenudson $M$ and Pitman MB: Endoscopic ultrasound-guided fine needle aspiration (EUS-FNA) contributes to a triple-negative test in preoperative screening of pancreatic cysts. Cancer Cytopathol 122: 412-419, 2014.

26. Wang Y, Yan K, Fan Z, Sun L, Wu W and Yang W: Contrastenhanced ultrasonography of pancreatic carcinoma: Correlation with pathologic findings. Ultrasound Med Biol 42: 891-898, 2016. 